\title{
FACTORES QUE DIFICULTAN LA RELACIÓN EDUCATIVA ENTRE LA EDUCACIÓN ESCOLAR Y EL SABER Y CONOCIMIENTO MAPUCHE
}

\author{
FACTORS THAT DIFFICULT THE EDUCATIONAL RELATIONSHIP BETWEEN \\ SCHOOL EDUCATION AND KNOWLEDGE MAPUCHE
}

\author{
Juan Carlos Beltrán Véliz* y Sergio Pérez Morales*
}

\begin{abstract}
Este estudio buscó describir los factores que dificultan la relación educativa entre la educación escolar y el saber y conocimiento mapuche, en escuelas situadas en contextos interculturales de la zona costera de La Araucanía. Se planteó un diseño cualitativo de nivel descriptivo, a base de un estudio de casos. Las técnicas de recolección de información fueron la entrevista semiestructurada y grupo focal. Se evidencia la presencia de un currículo monocultural, debido a la escasa incorporación de conocimientos de la cultura mapuche que están arraigados en el medio familiar. Asimismo, se visualizan prácticas de enseñanza basada en la cultura occidental anclada en el conductismo. Además, se aprecia ausencia de trabajo colaborativo debido a la falta de liderazgo de los directivos, porque sus funciones están centradas en aspectos administrativos, y control de actividades. Finalmente, se observa escasa generación de proyectos educativos para incluir a la comunidad mapuche en las actividades de la escuela. Se concluye que la escuela no constituye un espacio para articular los saberes escolares con los conocimientos y saberes de la cultura mapuche.

Palabras claves: Educación intercultural, currículum intercultural, conocimientos culturales mapuches, diálogo.
\end{abstract}

This study sought to describe the factors that impede the educational relationship between school education and Mapuche knowledge in schools located in intercultural contexts of the coastal area of La Araucanía. A qualitative design was proposed at the descriptive level, based on a case study. The data collection techniques used were semi-structured interviews and focus group. The evidence shows the presence of a monocultural curriculum, due to the scarce incorporation of Mapuche cultural knowledge rooted in the family environment. Likewise, teaching practices are based on western culture and anchored in behaviorism. In addition, there is absence of collaborative work due to the lack of leadership in school's board of directors, since their functions are focused on administrative aspects, and on the control of activities. Finally, there is little generation of educational projects to include the Mapuche community in the activities of the school. It is concluded that the school does not constitute a space to articulate school knowledge with the Mapuche cultural knowledge.

Key words: Intercultural education, intercultural curriculum, Mapuche cultural knowledge, dialogue.

\section{Introducción ${ }^{1}$}

El propósito de la escuela es la transmisión de un patrimonio cultural único donde históricamente la escuela, por su naturaleza homogeneizadora, ha entregado una educación monocultural a los alumnos de origen indígena, negando la inclusión y la racionalidad de sus saberes educativos (Quilaqueo, Fernández y Quintriqueo, 2010). Desde esta perspectiva, la educación en contextos de diversidad sociocultural se realiza basado en contenidos escolares expresados en concepciones e intereses que no responden a la sociedad que vive un estado de dominación sociopolítica, sociocultural y económica (Sarrasin, 1996; Forquin, 1997).
Desde el contexto mapuche, los niños recibieron una educación centrada en contenidos occidentales, finalidades educativas enmarcadas en la racionalidad positivista decimonónica y en segunda lengua (Durán y Catriquir, 2007; Schmelkes, 2009; Serrano, 2012). De acuerdo con lo expuesto, Durán y Ramos (1986) señalan que alrededor de 1819 surge una legislación que define al mapuche como chileno y lo que garantiza la posibilidad de educarse. Los mapuches son así objeto de una escolarización forzada, ahora legalmente instituida que se proponía enseñar a leer, escribir, y contar en castellano y con la prohibición factual de hablar la lengua materna. En correspondencia, en un estudio llevado a cabo por Quintriqueo y McGinity (2009), se evidencia

\footnotetext{
* Universidad Católica de Temuco, Facultad de Educación, Temuco, Chile. Correo electrónico: jbeltranucatolica@ gmail.com; sperez@uct.cl
} 
que cuando los niños/as se incorporan a la educación escolar adquieren un conjunto de contenidos y finalidades educativas, objetivos de enseñanza y aprendizaje, que se fundamentan en lógicas de saberes y conocimientos educativos no mapuches. $\mathrm{Al}$ respecto, estos autores observan que el 69,8\% de alumnos y $76 \%$ de los profesores declaran que existe una ausencia de la lengua mapuche en el medio escolar como mecanismo principal de transmisión de la memoria social mapuche con relación a saberes y conocimientos para la formación de personas. En este contexto, la escuela asume como política el desarrollo de una educación escolar para la población indígena, donde se explicita el castellano como lengua oficial y, por esta razón, la negación del mapuzungun como lengua de enseñanza escolar (Quintriqueo, 2010).

$\mathrm{Al}$ respecto, Quidel (2011), en una investigación, constata que las estrategias metodológicas aplicadas para la enseñanza del mapuzungun, no responden al sistema de educación mapuche. Se añade que el empleo de la metodología de enseñanza occidental no se ajusta a la del mapuche, porque en ella va incluida la visión de mundo de las culturas occidentales. Asimismo, Lara (2012) visualiza prácticas de enseñanza de mapuzungun con frecuencia demasiado transmisoras y reproductivas, enmarcadas en la cultura occidental, y asentadas en el enfoque conductista. En tal sentido, en un estudio realizado en Vilcún por Mellado y Chaucono (2016) concluyen que en el aula se observan prácticas de enseñanza tradicionales de corte occidental, centradas en el currículo prescrito, ignorando la cultura mapuche. Es posible señalar que los profesores que operan en contextos interculturales reproducen el conocimiento occidental sobre la base de prácticas de enseñanza tradicionales centradas en el profesor y por tanto descontextualizadas, pues excluyen elementos, saberes y formas de enseñanza propias de la cultura mapuche, lo que fragmenta la generación de aprendizajes significativos.

Lo expuesto anteriormente se refleja en que la educación escolar carece de una relación educativa con el saber y conocimiento mapuche para la formación de niños y adolescentes (Quintriqueo, 2010). Esto resulta preocupante para el desarrollo de una educación intercultural adecuada, ya que "la escuela acalla activamente a los estudiantes a través de ignorar sus historias, de encuadrarlos dentro de clases con expectativas mínimas y de negarse a proporcionarles conocimientos relevantes para ellos" (Giroux y Mclaren, 1998:81). Esto tiende a la simplificación y fragmentación del conocimiento, lo que causa incomprensión de la realidad e identidad de los sujetos y no permite dar respuestas a la complejidad e incertidumbre de la vida (Morin, 2004). Desde las evidencias empíricas y discusión teórica se planteó el siguiente objetivo: describir los factores que dificultan la relación educativa entre la educación escolar y el saber y conocimiento mapuche, en escuelas situadas en contextos interculturales de la zona costera de La Araucanía.

\section{Marco teórico}

\section{La interculturalidad desde un enfoque crítico}

Para aproximarse a la dimensión de interculturalidad desde un enfoque crítico, primero es preciso abordar el enfoque intercultural. Cuando se habla de enfoque intercultural, o de interculturalidad en general, nos referimos a una forma de posicionarnos en el mundo, de mirar la realidad que nos rodea por medio del prisma de la diversidad y la complejidad que nos caracteriza. Esto implica, entre otras cosas, considerar la diversidad como inherente al ser humano y no como un déficit que necesita compensarse (Malik y Ballesteros, 2015). Desde esta perspectiva, es necesario respetar, valorar y reconocer la diversidad como parte esencial del ser humano, desde su participación, construcción y desarrollo de una cultura. En este plano, la interculturalidad debería consistir en la construcción cotidiana de una cultura en la que todos se reconocieran, en la que cupieran y participaran todas las personas, basada en el diálogo (Pereda, 2004). En este sentido, en la interculturalidad cobra relevancia la interacción entre las propias culturas que habitan un espacio común, en un proceso dinámico de interacción social y cultural bajo un plano igualitario (Aguado 2011; Besalú, 2002). Esto favorece la interacción constante, en un plano de igualdad de individuos y grupos de distintas culturas desde el diálogo. En esta lógica, la interculturalidad mediante el diálogo considera las relaciones intersubjetivas con la finalidad de comprender las experiencias del Otro y, así, disponerse a conocerlo y aceptarlo sociocultural e históricamente (Quilaqueo, Quintriqueo y Cárdenas, 2005). De acuerdo con lo anterior, las culturas se desarrollan mediante 
la interacción, reconocimiento y la comprensión del Otro mediante un intercambio de experiencias, vivencias, conocimientos propios entre culturas basada en el diálogo. Desde esta perspectiva, es necesario avanzar hacia una interculturalidad crítica, transformadora, emancipadora e igualitaria (Tubino, 2004).

Desde este punto de vista, la interculturalidad se concibe como práctica contrahegemónica, enfocada en revertir la designación de algunos conocimientos como legítimos y universales y en detrimento de aquellos de los pueblos indígenas relacionados con la naturaleza, el territorio y la ancestralidad (Sartorello, 2016:126).

En este sentido, Walsh (2009) sostiene que la interculturalidad crítica debe ser entendida como una herramienta pedagógica, la que pone en cuestionamiento continuo la racialización, subalternización e inferiorización y sus patrones de poder, visibiliza maneras distintas de ser, vivir y saber, y busca el desarrollo y creación de comprensiones y condiciones que no solo articulan y hacen dialogar las diferencias en un marco de legitimidad, dignidad, igualdad, equidad y respeto, sino que también alientan la creación de modos "otros"2 de pensar, ser, estar, aprender, enseñar, soñar y vivir que cruzan fronteras. Esto implica una búsqueda de una postura de diálogo, reflexión crítica a base de formas de pensar, de conocer, vivir, actuar sobre la base del reconocimiento y comprensión del otro.

En este campo, Quintriqueo et al. (2016) señalan que en el contexto educativo chileno se debe pensar en la interculturalidad como un proyecto educativo a construir, el que surja desde los padres, madres, profesores, estudiantes y la comunidad educativa en general, con el fin de que se materialicen en políticas públicas educativas interculturales. Este énfasis en lo culturalmente propio representa el punto de partida para dialogar críticamente con lo ajeno (Sartorello, 2009), mediante un proceso de articulación y contrastación entre lo propio y lo ajeno (Bonfil, 1983). En este plano, la interculturalidad desde una perspectiva crítica queda abierta a la posibilidad de que la interacción entre los sujetos signifique el volver a mirarse para despojarse de prejuicios y estereotipos que pueden ser explícitos o implícitos (Quilaqueo y Torres, 2013).

\section{Enfoque educativo intercultural}

El enfoque educativo intercultural permite el estudio y la comprensión de personas pertenecientes a diferentes culturas, lo que implica construir un saber multipolarizado con el fin de superar la discriminación y el racismo (Essomaba, 2012). "Este enfoque se concibe como una alternativa que favorece y permite la relación de saberes y de conocimientos de los pueblos indígenas con el conocimiento escolar" (Quintriqueo, et al. 2016:13). La utilización de este enfoque consiste en abandonar el ámbito del razonamiento eurocéntrico en el que se nos ha formado por años bajo una unilinealidad para comprender el mundo (Quilaqueo, et al. 2014). En tal sentido, se debe romper con una forma de pensar occidental, con la finalidad de avanzar hacia un pensamiento sustentado en la comprensión de un mundo inclusivo y tolerante. El enfoque educativo intercultural surge como una propuesta pedagógica, donde la subjetividad no es concebida como un obstáculo o como atentado contra la cientificidad, sino como fuente de conocimientos, en la medida en que se reconoce el papel activo de los actores sociales que construyen su realidad (Quilaqueo y Torres, 2013). Este enfoque enfatiza los procesos e interacciones que unen y definen a los individuos y a los grupos poniendo énfasis en el diálogo que surge entre estos (Essomba, 1999), lo que favorecería la convivencia en un contexto intercultural, debido a que el aporte de culturas distintas, sustentado en el diálogo, constituye una de las fuentes imprescindibles para el enriquecimiento y la construcción de proyectos educativos y sociales en común.

\section{Currículum escolar intercultural}

Para González (2011) el currículum intercultural es un marco teórico-práctico que trata de dar respuesta a los conflictos detectados en contextos multiculturales, bajo el valor de la diversidad étnica, erradicando toda práctica discriminatoria, segregacionista o racista y aplicando métodos didácticos que están orientados a producir transformaciones sociales. Al respecto, Zabala (1997) señala que se debe considerar que los contenidos escolares requieren de elementos socioculturales propios del entorno del alumno. Desde esta mirada, deben estar presentes saberes y conocimientos mapuches en el currículum escolar. En tal sentido, el enfoque educativo intercultural se propone lograr una 
apertura epistemológica del conocimiento escolar (Quintriqueo, et al. 2016). Esta apertura se puede dar desde una relación sustentada en un currículum escolar intercultural, el que favorecería la articulación educativa entre aquellos saberes y conocimientos institucionalizados en el sistema educativo y los saberes propios de la comunidad mapuche, como un conjunto de contenidos, intereses y finalidades deseables de incorporar en los procesos educativos escolares (Quintriqueo y McGinity, 2009). Esto permitiría reconocer los saberes y conocimientos mapuches y, por tanto, debiesen estar en diálogo constante para poder respetarlos e incorporarlos a la educación de todos los estudiantes, mapuches y no mapuches (Quintriqueo, et al. 2016). Lo anterior favorecería el diálogo entre los actores de la escuela con los miembros de la comunidad mapuche, con el fin de intercambiar saberes y conocimientos mapuches y escolares, y contribuir a una educación inclusiva y de calidad en contextos educativos interculturales.

\section{Metodología}

El estudio es cualitativo, pues propone una forma de construcción del conocimiento, basándose en la subjetividad e intersubjetividad, en el contexto en que ocurren los fenómenos se sirve de la descripción e interpretación para la comprensión de fenómenos socioculturales (Bartolomé, 1992; Sandín, 2003; Valles, 2007). El nivel es descriptivo denso, esto implica desentrañar los significados que surgen del contenido verbal de los sujetos, en un intento de preservar su riqueza lo más fielmente posible (Geertz, 1973). El diseño corresponde al estudio de casos, el que está "caracterizado por el examen sistemático y en profundidad de casos de un fenómeno, entendiendo estos como entidades sociales o entidades educativas" (Bisquerra, et al. 2012:309).

El caso está constituido por 35 participantes: 15 profesores de educación intercultural bilingüe y 20 profesores occidentales ${ }^{3}$ de escuelas subvencionadas y municipales del sector rural situadas en contextos interculturales de la zona costera de La Araucanía. La selección de los sujetos fue intencionada, en este sentido se debe "seleccionar a quienes tengan un mejor conocimiento del fenómeno por investigar. Esto garantiza una saturación efectiva y eficiente de las categorías con información de óptima calidad" (Gurdián, 2007:247). Las técnicas de recolección de datos que se aplicaron fueron la entrevista semiestructurada y grupo focal.

\section{Análisis de datos}

Los datos que emergieron de las entrevistas semiestructuradas y de los grupos focales fueron analizados con el programa Atlas ti 7.0. En una primera fase fueron reducidos mediante el proceso de codificación abierta. Al respecto, Strauss y Corbin (2002) señalan que para descubrir y desarrollar los conceptos se debe abrir el texto y exponer los pensamientos, ideas y significados contenidos en este. Durante el proceso de codificación abierta se levantaron las categorías desde una lógica inductiva, "sumergiéndose literalmente en el documento o situación para identificar los temas o las dimensiones más relevantes" (Bisquerra, et al. 2012). Estas categorías fueron etiquetadas, examinadas y comparadas en términos de sus similitudes y diferencias, al amparo de una aproximación sustantiva a la realidad, priorizando para su levantamiento la intensidad del relato, por sobre la frecuencia de ideas en el mismo (Flick, 2007). En una segunda fase se realizó el proceso de codificación axial, entendiéndose el acto en el que las categorías se interrelacionan y se vinculan (Strauss y Corbin, 2002). Posteriormente se llevó a cabo una comparación de las categorías con los referentes del marco teórico, para confrontarlas con los fragmentos de las entrevistas semiestructuradas y grupos focales a partir de lo que se denomina muestreo teórico-empírico mediante el empleo del Método Comparativo Constante (MCC). Este procedimiento permitió desarrollar la triangulación de datos, y finalmente se realizó la discusión de resultados por categoría.

\section{Discusión de resultados}

Desde la codificación abierta y axial emergieron las siguientes categorías: 1) Currículum monocultural, 2) Conocimientos de la cultura mapuche, 3) Enseñanza monocultural y conductista 4) Liderazgo escolar, 5) Trabajo pedagógico colaborativo, y 6) Proyectos educativos.

\section{Categoría 1. Currículum monocultural}

En esta categoría se presenta el currículum monocultural como una de las limitantes, ya que obstaculiza la incorporación de conocimientos 
mapuches. Esto se refleja en la siguiente textualidad: "La educación siempre ha sido homogenizante, los conocimientos que se entregan son de la cultura occidental, salvo los conocimientos que se entregan en la signatura de la lengua mapuche, pero son escasos, y en las demás asignaturas no se integran" (E2:P3). En continuidad se aprecia el siguiente relato: "En el caso de ciencias naturales, no están contemplados los conocimientos de la cultura mapuche. Sería muy importante incorporarlos, porque a los niños mapuches se les haría más significativo aprender, y tendrían otra forma de ver las cosas" (E6:P12). Otro entrevistado relata: "Lamentablemente con la historia estamos súper mal para los niños, ejemplo: todavía se enseña como descubrimiento de América, y no se habla que realmente fue una invasión. Yo debería enseñar también la historia del pueblo mapuche y sus costumbres, tradiciones, etc." (E22:P2).

Respecto de tales evidencias, si bien es cierto se incorporaron ciertos saberes y finalidades educativas en la lengua mapudungun, no obstante en las asignaturas tradicionales ${ }^{4}$ no se incluyen, además se evidencia una enseñanza de contenidos apartada de la realidad. Esto hace complejo el proceso de aprendizaje y por tanto la formación de los estudiantes mapuches que están insertos en contextos interculturales. En este sentido, Quintriqueo y McGinity (2009) señalan que históricamente la escolarización de niños/as mapuches se ha realizado sobre la base de contenidos y finalidades educativas monoculturales occidentales. Situación que hasta hoy sigue siendo una realidad (Mansilla, Becerra y Merino, 2015). En contraste, Quintriqueo, Torres, Gutiérrez, y Sáez (2011) constatan que los estudiantes aprenden en la diferencia, considerando el conocimiento indígena y no indígena para mejorar la calidad de los aprendizajes, oportunidades y convivencia social. En tal sentido, se deben incluir en el currículo escolar las racionalidades de la cultura mapuche. Esto enriquecería la educación de niños mapuches y no mapuches.

\section{Categoría 2. Conocimientos de la cultura mapuche}

En esta categoría se aprecia la escasa incorporación de conocimientos de la cultura mapuche desde la familia en la educación escolar; esto queda

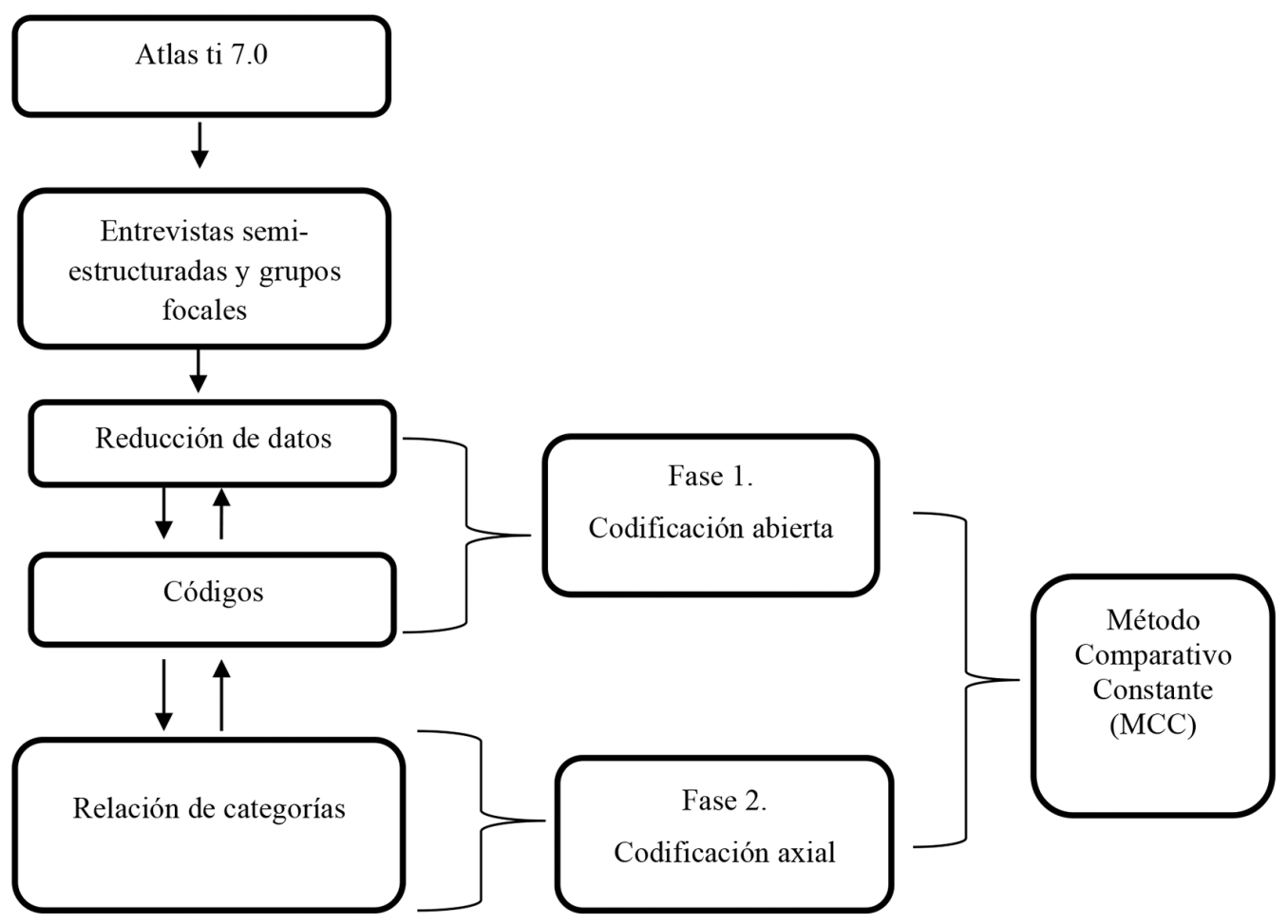

Figura 1.Utilización del atlas ti 7.0, en el proceso de codificación abierta y axial. 
de manifiesto en la siguiente textualidad: "Es necesario integrar la familia en la escuela, ya que es en la familia donde se le enseña al niño mapuche su cultura" (E18:P3). Al respecto, un profesor intercultural declara: "Los conocimientos, valores, saberes, creencias, tradiciones, se han enseñado de abuelo a padre y padre a hijo. Son muy importantes para la formación de los niños y jóvenes en la familia, por lo que debieran ser incorporados en la escuela" (E7: P10).

Respecto de tales evidencias, Quintriqueo y Torres (2013) constata que en las escuelas establecidas en contexto mapuche, la formación de niños y jóvenes se ha llevado a cabo a base de saberes muy distantes de los que se enseñan en el medio familiar mapuche. La escasa incorporación de conocimientos culturales que están arraigados en el medio familiar, obstaculiza la formación de los jóvenes mapuches y por tanto su identidad se ve afectada. Esto ha provocado una disminución o exclusión del uso de los saberes educativos mapuches, provocando en muchos casos en los jóvenes la autonegación de la identidad mapuche (Quintriqueo y Maheux, 2004). Esto induciría a la pérdida de la identidad de los niños y jóvenes mapuches, por tanto su cultura.

\section{Categoría 3. Enseñanza monocultural y conductista}

En esta categoría es posible evidenciar una enseñanza monocultural centrada en el conductismo. Esto se refleja en lo consecutivo: "Utilizo bastante la repetición de palabras y con vocabulario de lengua mapuche, por decirte, les doy cierta cantidad de términos para que ellos los busquen en el diccionario" (E5:P8). Asimismo, otro entrevistado declara: "Yo trabajo con ellos con lectura, ejemplo les digo: "Abran su libro de mapudungun, primero leeré yo en mapudungun luego ustedes repiten. Cuando terminamos la lectura ellos buscan las palabras que no entiendan en el diccionario de mapudungun" (E11:P3). Al respecto, las formas de enseñanza que emplean la mayoría de los profesores de la asignatura de mapuzungun, están centradas en la cultura occidental con marcados énfasis en el paradigma conductista. En tal sentido, en un estudio realizado por Mellado y Chaucono (2015) en el contexto mapuche, evidencian que las prácticas de aula predomina un enfoque conductista, situación recurrente en casi todas las clases observadas. Lo expuesto se robustece por una investigación realizada por Quidel (2011), quien evidencia que los alumnos realizan la lectura de textos en grupo de manera repetitiva. En tal sentido, afirma que la finalidad de la lectura repetitiva es para que los alumnos vayan internalizando los sonidos y las palabras en mapuzungun en forma mecánica, sin existir una lectura comprensiva del texto. Además, constata que las estrategias metodológicas aplicadas para la enseñanza del mapuzungun en el Programa de Educación Intercultural Bilingüe (en adelante PEIB) no responden al sistema de educación mapuche. Esto, porque la enseñanza de la lengua se realiza mediante las características que son propias y específicas de la cultura occidental.

De igual manera, Williamson y Flores (2015) señalan que el PEIB no favorece la introducción de las lógicas de enseñanza indígenas dentro de la escuela y no contribuye a mejorar la calidad de la enseñanza. En este contexto, en las asignaturas tradicionales una profesora relata:

En mis clases de lenguaje y comunicación, yo no empleo formas de enseñanza de la cultura mapuche, porque no las conozco y, no hay metodologías de enseñanza mapuche en los programas de lenguaje. Te digo que es necesario enseñar empleando estrategias metodológicas de la cultura mapuche, porque así los niños tienen diversas formas de aprender y de ver la realidad (E32:P7).

Otro profesor relata: "En ciencias naturales sería muy significativo enseñar empleando metodologías de la cultura mapuche, esto porque ellos están muy ligados a la naturaleza, a la tierra. Sería muy bueno que nos capacitaran sobre métodos de enseñanza del pueblo mapuche" (E4:P3). Referente a las narraciones de profesores, que enseñan en las asignaturas tradicionales, no se visualiza la aplicación de formas de enseñanza de la cultura mapuche porque los profesores las desconocen. Además, los entrevistados señalan que es necesario emplear metodologías de enseñanza propias de la cultura mapuche, con el fin de dar sentido a la enseñanza $y$, en consecuencia, favorecería la adquisición de aprendizajes significativos. En tal sentido, los sujetos relatan que es necesario asistir a capacitaciones acerca de métodos de enseñanza de la cultura mapuche. En correspondencia, para que el diseño de la enseñanza sea pertinente Quintriqueo, Gutiérrez, Torres y Sáez (2011) proponen una metodológica 
intercultural en la que las lógicas de conocimiento tanto occidental como indígena se plasmen en la planificación de la acción didáctica, sobre la base de objetivos, recursos y estrategias didácticas que generan la relación dialógica de saberes educativos.

\section{Categoría 4. Liderazgo escolar}

Esta categoría presenta una ausencia de liderazgo escolar emanado desde el director y jefes de unidades técnicas pedagógicas para establecer una relación educativa entre la escuela y la comunidad mapuche. En este contexto, un profesor manifiesta: "No, en la escuela no se ve un liderazgo, los directivos más bien se dedican a administrar y controlar nuestras actividades. No se conforman equipos de trabajo con los profesores, para establecer una relación con la comunidad mapuche" (17:P8). Un tercer entrevistado relata: “¿Liderazgo?, No, no hay liderazgo, porque no existe una buena organización, colaboración de parte del director y también del jefe de la unidad técnica pedagógica para generar actividades que integren a la comunidad en las actividades escolares" (E14:P3). Lo anterior se robustece por lo constatado por Chaucono, Mellado y Yuste (2017), donde muestran que el 70\% del directivo desarrolla su gestión desde una mirada que restringe el contexto rural mapuche, la reciprocidad entre cultura occidental e intercultural.

Se puede apreciar una falta de liderazgo escolar por parte de los directivos, ya que se observa falta de organización y creación de equipos de trabajo para desarrollar actividades colaborativas que permitan establecer una relación educativa adecuada entre la escuela y la comunidad mapuche, lo que limita el diálogo entre conocimientos culturales mapuches $\mathrm{y}$ conocimientos escolares $\mathrm{y}$, por tanto, restringe una educación intercultural. Esto se debe a que las funciones de los directivos están focalizadas en aspectos administrativos, y control de actividades. Al respecto, Sepúlveda (2005) sostiene que el control burocrático está centrado en las instrucciones, en los requerimientos, formularios, solicitudes de informes a los profesores. En tanto, la función de los directores debería estar centrado en el liderazgo escolar, pues permite "movilizar e influenciar a otros para articular y lograr las intenciones y metas compartidas de la escuela" (Leithwood, 2009:20). En este sentido, este autor señala que el dinamizador y generador de los procesos de gestión de un establecimiento es el liderazgo, ejercido principalmente por los equipos directivos y de gestión de cada establecimiento. Entonces, los directivos deben disponer de la capacidad de influenciar y movilizar al equipo de profesores para desarrollar actividades educativas en conjunto con la comunidad mapuche, con el objetivo de fortalecer la inclusión y articulación de saberes y conocimientos mapuches y escolares.

\section{Categoría 5. Trabajo pedagógico colaborativo}

Se presenta ausencia de trabajo colaborativo entre profesores respecto de la articulación pedagógica entre asignaturas: "La única articulación... trabajo colaborativo, que se hace es cuando se celebra el we tripantu (año nuevo mapuche) y de ahí cada uno se va a su asignatura, pero no nos reunimos periódicamente para intercambiar planificaciones, ideas y estrategias didácticas" (E13:P8). De acuerdo con Hargreaves, Earl, Ryan (2002: 271), “...los profesores han trabajado en buena medida aislados". Lo anterior se refleja en el la siguiente narración:

Hee... más allá de hablar algunas cosas educativas en el pasillo, no se generan los espacios para trabajar de forma colaborativa, para intercambiar experiencias entre los profesores de todas las asignaturas. No hay espacios para trabajar. Yo como profesora de historia y los demás profesores nunca nos hemos reunido con la profesora de mapudungun para articular contenidos, compartir metodologías de enseñanza, recursos didácticos, etc. (E14:P7).

De las narraciones expuestas se puede visualizar ausencia de espacios para desarrollar un trabajo colaborativo entre profesores de las distintas asignaturas, con el fin de articular conocimientos, intercambiar experiencias, ideas, planificaciones, recursos didácticos, y estrategias didácticas. Al respecto, Little (1990) señala que los profesores apenas han compartido entre ellos recursos e ideas, raras veces han visitado las aulas de los demás, y solo muy ocasionalmente han participado en la planificación o en la resolución conjunta de problemas. La ausencia del trabajo pedagógico colaborativo dificulta el desarrollo de clases enriquecedoras y contextualizadas. Al respecto, Delgado y Solano (2009) señalan que el trabajo colaborativo pretende la construcción de conocimiento en equipo, empleando estructuras de comunicación y 
de colaboración. Entonces, el trabajo colaborativo permitiría enriquecer las prácticas educativas de los profesores y por tanto contribuir a la adquisición de las habilidades, capacidades y competencias por parte de los educandos.

\section{Categoría 6. Proyectos educativos}

Esta categoría da cuenta de la escasa generación de proyectos educativos por parte de la escuela para incluir a la comunidad mapuche, tal afirmación se evidencia en la siguiente textualidad: "En la escuela se realiza un proyecto de integración, el we tripantu, pero no se realizan otros proyectos educativos que involucren a los mapuches realmente. Entonces se debe integrar a la familia mapuche y no mapuche, ya que es un agente importante en la formación de los alumnos" (E16:P8). Otra entrevistada relata:

No hay proyectos, es una cosa mal hecha, si yo trabajo en esta escuela, lo ideal sería que esta escuela estuviera participando con la comunidad mapuche más cercana, tuviera un vínculo, un diálogo con las autoridades tradicionales, por ejemplo visitar, un Lonko, una Machi y también, integrarlos a la escuela, para intercambiar conocimientos (E19:P15).

De acuerdo con estas evidencias, se presenta solo el we tripantu como proyecto educativo. En consecuencia, los participantes relatan que la escuela debiera participar en proyectos, en conjunto con las comunidades mapuches. Asimismo, declaran que se debería establecer un vínculo anclado en diálogo con las autoridades de las comunidades mapuches para intercambiar conocimientos. En este sentido, se observa la necesidad de integrar a las autoridades de las comunidades mapuches y a la familia mapuche y no mapuche en la educación escolar, como agentes fundamentales en la formación de los estudiantes. Por tanto, se debe apostar por un proyecto de interculturalidad en educación que aborde no solo el reconocimiento de culturas indígenas, sino que busque construir un diálogo entre miembros de la sociedad indígena y no indígena (Quintriqueo, et al. 2016). Referente a tal afirmación, la escuela debiera sustentar los proyectos sobre la base del diálogo intercultural, pues supone una contextualización de ambas concepciones epistemológicas basadas en los contenidos y las finalidades educativas para la formación de personas en el medio educativo (Quintriqueo y Torres, 2013). En este sentido, el conocimiento del Otro constituye una de las fuentes imprescindibles (Pech, Rizo y Romeu, 2008) para la construcción de proyectos de acción en común en contextos de interculturalidad (San Martín y Quilaqueo, 2012). En consecuencia, la generación de proyectos educativos posibilitaría la formación adecuada de niños y jóvenes mapuches y no mapuches, asimismo, mejoraría la convivencia en contextos interculturales.

\section{Conclusión}

A partir de la discusión de resultados, es posible dar respuesta al objetivo propuesto para este trabajo. Desde las categorías desplegadas es posible constatar que: se presenta un currículo monocultural y, por tanto, descontextualizado como una de las limitantes substanciales, debido a la escaza incorporación de saberes y conocimientos en la asignatura lengua de mapuzungun y nula inclusión de estos en las asignaturas tradicionales. En tal sentido, se devela la escasa inclusión de saberes y conocimientos que están arraigados en el medio familiar, lo que obstaculiza la formación de los jóvenes mapuches, y por consiguiente su identidad y cultura se ven afectadas. En esta lógica, se aprecia un currículum prescrito fundamentado en una racionalidad positivista de corte occidental. Al respecto, Rodrigo y Arnay (1997) señalan que desde el positivismo lógico se hace hincapié en que solo es posible una clase de conocimiento: el conocimiento científico occidental. Para revertir esto es necesario incluir conocimientos de la cultura mapuche desde la familia al currículum escolar de manera transversal, para ello la escuela debe integrar e incluir a las familias mapuches y generar en conjunto un proyecto curricular intercultural, con la finalidad de ampliar el conocimiento del profesor y de los estudiantes, a partir de la relación entre el conocimiento mapuche y el conocimiento escolar. En tal sentido, permitiría enriquecer la educación desde un plano intercultural.

En este contexto, emergen las prácticas de enseñanzas monoculturales y conductistas, basadas en conocimientos y estrategias metodológicas occidentales implementadas por la mayoría de los profesores de la asignatura de mapuzungun. Asimismo, los profesores de las asignaturas tradicionales declaran 
el desconocimiento cabal de formas de enseñanza mapuche, es decir, se evidencia falta de un dominio del Conocimiento Didáctico del Contenido (CDC) (Shulman, 2005), lo que no contribuye a mejorar la calidad de enseñanza. En consecuencia, la enseñanza está centrada en la cultura occidental con marcados énfasis en el paradigma conductista. Para revertir lo anterior, Williamson y Flores (2015) sostienen que se debe conocer y experimentar la pedagogía y didáctica mapuche y no mapuche interculturalmente que se aplica y se puede utilizar en los espacios y territorios educativos. En este sentido, los profesores señalan que es necesario y adecuado emplear metodologías de enseñanza propias de la cultura mapuche, con el fin de dar sentido a la enseñanza y en consecuencia favorecería la adquisición de aprendizajes significativos, y por tanto contextualizados. En efecto, los profesores manifiestan la necesidad de capacitarse acerca de métodos de enseñanza de la cultura mapuche. En este sentido la escuela debe generar acciones encaminadas a que todos los profesores se capaciten en procesos y métodos de enseñanza y aprendizajes arraigados en la cultura mapuche. Asimismo, renovar sus prácticas de enseñanzas desde un plano diverso e inclusivo, donde se fomente el desarrollo de la reflexión críti$\mathrm{ca}$, trabajo colaborativo, el pensamiento divergente y la creatividad. Esto contribuiría a mejorar sus procesos didácticos y pedagógicos y, por tanto, los aprendizajes de los estudiantes situados en contextos culturales mapuches. Además permitiría formar personas desde una perspectiva integral.

Otro factor relevante es la ausencia de liderazgo escolar por parte del director y jefes de unidades técnicas pedagógicas, observándose falta de organización y creación de equipos de trabajo para desarrollar actividades que permitan establecer una relación educativa adecuada entre la escuela y la comunidad mapuche. Esto se debe a que sus funciones están centradas en aspectos administrativos, y control de actividades. En este contexto, se revela la ausencia de trabajo colaborativo entre profesores, en cuanto a la articulación de conocimientos e intercambio de experiencias educativas, ideas, planificaciones, estrategias y recursos didácticos desde la racionalidad mapuche y occidental. Respecto de lo anterior, es preciso que los directivos se capaciten en el ámbito de liderazgo, para desarrollar la capacidad de influenciar, movilizar y motivar a los profesores en sus diversas actividades educativas. Así como también coordinar las acciones educativas, generar espacios para desarrollar el trabajo pedagógico colaborativo en conjunto con los profesores y entre estos últimos. Esto, con la finalidad de generar actividades educativas en conjunto con la comunidad mapuche, asimismo, favorecer la articulación de saberes y conocimientos mapuches y escolares, encaminado a mejorar la formación de niños mapuches y no mapuches.

Finalmente, aparece como un obstaculizador relevante, la escasa generación de proyectos educativos para incluir a la comunidad mapuche en las actividades de la escuela. En tal sentido, los participantes relatan que la escuela debiera participar en conjunto con la comunidad mapuche mediante la colaboración sustentada en el diálogo, para intercambiar conocimientos. Para ello es necesario incorporar a las autoridades de las comunidades y familias mapuches y no mapuches en la educación escolar como agentes importantes en la formación de niños y jóvenes, esto mediante la colaboración y diálogo. Al respecto, Pech, Rizo y Romeu (2008) sostienen que para dialogar y convivir en un contexto intercultural, el conocimiento del otro constituye uno de los elementos imprescindibles para establecer una comunicación intercultural, como el puente necesario para propiciar proyectos de vida compartidos por las culturas implicadas. En este sentido, la escuela debe generar proyectos educativos interculturales con la finalidad de integrar e incluir a los distintos miembros de la comunidad mapuche y no mapuche como agentes fundamentales en la construcción de una educación inclusiva, equitativa, justa e integral. Además, esto favorecería a la creación de proyectos de vida en común entre la escuela y las comunidades mapuches y, en consecuencia, permitiría fortalecer la convivencia social entre miembros de estas.

En relación con las conclusiones y las propuestas, la escuela debe constituirse en un espacio para una educación intercultural mediante la inclusión de saberes, conocimientos culturales mapuches y para la contextualización de las prácticas de enseñanza de los profesores. Además, debe favorecer a la articulación entre el conocimiento científico occidental y mapuche. Para ello se precisa de un liderazgo centrado en el trabajo pedagógico colaborativo, para construir proyectos educativos sustentados en el diálogo, con el fin de contribuir y enriquecer a la formación de niños y jóvenes mapuches y no mapuches desde un plano integral. Así como también favorecer las relaciones sociales de personas que conviven e interactúan en contextos educativos interculturales. 


\section{Agradecimientos}

Expreso mis agradecimientos a la Universidad de La Frontera de Temuco, Chile, y a la Facultad de
Educación de la Universidad Católica de Temuco, Chile. De igual forma, agradezco a los participantes que contribuyeron con información para el desarrollo de este estudio.

\section{Referencias Citadas}

Aguado, T.

2011 "El enfoque intercultural en la búsqueda de buenas prácticas escolares". Revista Latinoamericana de Educación Inclusiva, Vol. $5 \mathrm{~N}^{\circ}$ 2: 23-42.

Bartolomé, M.

1992 "Investigación cualitativa en Educación: ¿Comprender o transformar?", Revista de Investigación Educativa, $\mathrm{N}^{\circ} 20$, 7-36, Universidad de Murcia, España.

Besalú, X.

2002 Diversidad cultural y educación. Síntesis, Madrid, España.

Bisquerra, R.; Dorio, I.; Gómez, J.; Latorre, A.; Martínez, F.;

Massot, I.; Mateo, J.; (...) Vilá, R.

2012 Metodología de la investigación educativa. La Muralla S.A, Madrid, España.

Bonfil, G.

1983 "Lo propio y lo ajeno: una aproximación al problema del control cultural". En Educación, etnias y descolonización en América Latina: una guía para la educación bilingüe intercultural, N. Rodríguez, E. Masferrer, R. Vargas, pp. 249256. UNESCO, México D.F., México.

Chaucono, J.; Mellado, M. y Yuste, R.

2017 Creencias de directivos escolares sobre educación intercultural de escuelas rurales en contexto mapuche. file://C:/Users/beltr/Downloads/031_Chaucono.pdf (22 de enero 2018).

Delgado, M. y Solano, A.

2009 "Estrategias didácticas creativas en entornos virtuales para el aprendizaje". Revista Electrónica Actualidades Investigativas en Educación, Vol. 9, $\mathrm{N}^{\circ}$ 2: 1-21, Universidad de Costa Rica, San José, Costa Rica.

Durán, T. y Catriquir, D.

2007 "Kimeltuwün zugu. Modelo educativo mapuche". En Patrimonio cultural mapuche: acercamientos metodológicos e interdisciplinarios derechos lingüísticos, culturales y sociales, T. Durán, D. Catriquir, A. Hernández, pp. 443-454. Universidad Católica de Temuco, Chile.

Durán, T. y Ramos, N.

1986 "El papel de algunos fenómenos sociolingüísticos en procesos socioculturales de cambio entre los mapuches". Cultura, Hombre, Sociedad, Vol. 3 N 2: 409-419.

Essomba, M.

1999 Construir la escuela intercultural. Reflexiones y propuestas para trabajar la diversidad étnica cultural. Editorial Graó, Barcelona, España.

Essomba, M.

2012 Inmigración e interculturalidad en la ciudad. Principios, ámbitos y condiciones para una acción comunitaria intercultural en perspectiva europea. Graó, Barcelona, España.

Flick, U.

2007 Introducción a la investigación cualitativa. Morata, S.L. Madrid, España.
Forquin, J.

1997 Les sociologues de l'éducation américains et britanniques. Présentation et choix de textes. De Boeck Université, Bruxelles.

Geertz, C.

1973 La interpretación de las culturas. Gedisa, Barcelona, España.

Giroux, H. y Mclaren, P.

1998 Sociedad, cultura y educación. Miño y Dávila, Madrid, España.

González, E.

2011 La interculturalidad: implicaciones educativas. Actas del I Congreso Internacional sobre Migraciones en Andalucía, pp. 697-702. Universidad de Granada, España.

Gurdián, A.

2007 El paradigma cualitativo en la investigación socioeducativa. Print Center, San José, Costa Rica.

Hargreaves, A. Earl; L. Ryan, J.

2002 Una educación para el cambio. Reinventar la educación de los adolescentes. Ediciones Octaedro, S.L, Barcelona, España.

Lara, M.

2012 Aprender a leer y a escribir en la lengua mapudungun, como elemento de recuperación y promoción de la cultura mapuche. Tesis doctoral, Universidad Autónoma de Barcelona. Barcelona, España.

Leithwood, K.

2009 ¿Cómo liderar nuestras escuelas? Aportes desde la investigación. Salesianos Impresores, Santiago, Chile.

Little, J.

1990 "The persistence of privacy: Autonomy and initiative in teachers' professional relations"'. Teachers College Record, Vol. 9, No 4: 509-536. Columbia University New York.

Malik, B. y Ballesteros, B.

2015 "Construcción del conocimiento desde el enfoque intercultural". Diálogo Andino, № 47: 15-25.

Mansilla, J.; Becerra, S. y Merino, M.

2015 "Curriculum Violence: Occidental knowledge hegemony in relation to indigenous knowledge". Procedia-Social and Behavioral Sciences, Vol. 190, 434-439, University of Cologne, Germany.

Mellado, M. y Chaucono, J.

2015 "Creencias pedagógicas del profesorado de una escuela rural en el contexto mapuche". Revista Actualidades Investigativas en Educación. Vol. 15, № 3: 1-19.

Mellado, M. y Chaucono J.

2016 "Liderazgo pedagógico para reestructurar creencias docentes y mejorar prácticas de aula en contexto mapuche". Revista Electrónica Educare, Vol. 20, № 1: 1-18. Universidad de Costa Rica, San José, Costa Rica.

Morin, E.

2004 La mente bien ordenada. Repensar la reforma. Reformar el pensamiento. Editorial Seix Barral. Barcelona, España. 
Pereda, C.

2004 Los desafios de lo intercultural. I Encuentro Internacional sobre filosofía de la cultura y multiculturalismo, pp. 37-54. Campeche: INAH, Conaculta, Universidad Autónoma de Campeche.

Pech, C.; Rizo, M. y Romeu, V.

2008 Manual de comunicación intercultural. Universidad Nacional Autónoma de México. México, D.F.

Quidel, G.

2011 "Estrategias de enseñanza de la lengua mapunzugun en el marco del PEIB Mineduc-Origenes". Cuadernos Interculturales, Vol. 9, $\mathrm{N}^{\circ}$ 16: 61-80. Universidad de Playa Ancha, Valparaíso.

Quilaqueo, D.; Quintriqueo, S. y Cárdenas, P.

2005 Educación, currículum e interculturalidad. Elementos sobre formación de profesores en contexto mapuche. Frasis editores, Universidad Católica de Temuco, Chile.

Quilaqueo, D.; Fernández, C. y Quintriqueo, S.

2010 Interculturalidad en contexto mapuche. Editorial de la Universidad Nacional del Comahue, Neuquén, Argentina.

Quilaqueo, D. y Torres, H.

2013 "Multiculturalidad e interculturalidad: desafíos epistemológicos de la escolarización desarrollada en contextos indígenas". Alpha, No 37: 285-300, Universidad de Concepción, Chile.

Quilaqueo, D.; Quintriqueo, S.; Torres, H. y Muñoz, G.

2014 "Saberes educativos mapuches: aportes epistémicos para un enfoque de educación intercultural". Chungará, Vol. 46, № 2: 271-283, Universidad de Tarapacá. Arica, Chile.

Quintriqueo, S. y Maheux, G.

2004 "Exploración del conocimiento sobre la relación de parentesco como contenido educativo para un currículum escolar intercultural en comunidades mapuche". Revista de Psicología, Vol. 13, № 1: 73-91, Universidad de Chile.

Quintriqueo, S. y McGinity, M.

2009 "Implicancias de un modelo curricular monocultural en la construcción de la identidad sociocultural de alumnos/as mapuches de la IX región de La Araucanía, Chile”. Estudios Pedagógicos, Vol. 35, № 2: 173-188.Universidad Austral de Chile, Valdivia.

Quintriqueo, S.

2010 Implicancias de un modelo curricular monocultural en contexto mapuche. Gráfica LOM, Santiago, Chile.

Quintriqueo, S.; Torres, H.; Gutiérrez, M. y Sáez, D.

2011 "Articulación entre el conocimiento cultural mapuche y el conocimiento escolar en ciencia". Educación y Educadores, Vol. 14, No 3: 475-492, Universidad de la Sabana, Colombia.

Quintriqueo, S. y Torres, H.

2013 "Construcción de conocimiento mapuche y su relación con el conocimiento escolar". Estudios Pedagógicos, Vol. 39, $N^{\circ}$ 1: 199-216, Universidad Austral de Chile, Valdivia, Chile.

Quintriqueo, S.; Morales, S.; Quilaqueo, D. y Arias, K.

2016 Interculturalidad para la formación inicial docente: Desafíos para construir un diálogo intercultural, Ediciones Universidad Católica de Temuco, Chile.

Rodrigo, M. y Arnay, J.

1997 La construcción del conocimiento escolar. Paidós, Barcelona.

Sandín, M.

2003 Investigación cualitativa en educación. Fundamentos y tradiciones. McGraw-Hill, Madrid, España.
San Martín, D. y Quilaqueo, D.

2012 "Habitus profesional y relaciones intersubjetivas entre profesores principiantes y experimentados". Perfiles Educativos, Vol. 34, No 136: 63-78. Universidad Nacional Autónoma de México. México, D.F.

Sarrasin, R.

1996 "Bilinguisme et biculturalisme chez les Atikamekw". Revue canadienne de l'éducation, Vol. 19 No 2: 165-181, Université de Toronto, Canadá.

Sartorello, S.

2009 "Una perspectiva crítica sobre interculturalidad y educación inter-cultural bilingüe: El caso de la Unión de Maestros de la Nueva Educación para México (UNEM) y educadores independientes en Chiapas". Revista latinoamericana de educación inclusiva, Vol. 3, № 2: 77-87. Universidad Central de Chile, Santiago, Chile.

Sartorello, E.

2016 La co-terización intercultural de un modelo educativo en Chiapas. Abya-Yala, Quito, Ecuador.

Serrano, $\mathrm{S}$.

2012 Historia de la educación en Chile (1810-2010). Tomo II La educación nacional (1880-1930). Taurus, Santiago, Chile.

Sepúlveda, G.

2005 La coordinación pedagógica. Grupo Innovat, Universidad de la Frontera, Temuco, Chile.

Schmelkes, S.

2009 "Interculturalidad, democracia y formación valoral en México". Revista Electrónica de Investigación Educativa, Vol. 11, Nº 2:1-10, Universidad Autónoma de Baja California, Ensenada, México.

Shulman, L.

2005 "Conocimiento y enseñanza: fundamento de la nueva reforma”. Profesorado. Revista de Currículum y Formación de Profesorado, Vol. 9, № 2: 1-30, Universidad de Granada, España.

Tubino, F.

2004 "Del interculturalismo funcional al interculturalismo crítico". En M. Samaniego y C. Garbarini (Eds.), Rostros y fronteras de la identidad. Universidad Católica de Temuco, Chile.

Strauss, A. y Corbin, J.

2002 Bases de la investigación cualitativa. Técnicas y procedimientos para desarrollar la teoría fundamentada. Universidad de Antioquia, Medellín, Colombia.

Valles, M.

2007 Técnicas cualitativas de investigación social. Reflexión metodológica y práctica profesional. Síntesis, Madrid, España.

Walsh, C.

2009 Interculturalidad, Estado, Sociedad: Luchas (de) coloniales de nuestra época. Ediciones Abya-Yala, Universidad Andina Simón Bolívar, Quito, Ecuador.

Williamson, G. y Flores, F.

2015 Estado del arte de la Educación Intercultural bilingüe en Chile, 1990-2013. Colección Espiral Social, Universidad de la Frontera de Temuco, Chile.

Zabala, A.

1997 "Los enfoques didácticos". En El constructivismo en el aula, C. Coll, E. Martín, T. Mauri, M. Miras, J. Onrubias, I. y Solé, pp. 125-161. Graó, Barcelona, España. 


\section{Notas}

1 Forma parte de los hallazgos de la tesis doctoral: Orientaciones didácticas para desarrollar de modo pertinente la enseñanza en profesores de educación general básica en escuelas situadas en contextos interculturales de la zona costera de La Araucanía.

2 Hablar de modos "otros" es tomar distancia de las formas de pensar, saber, ser y vivir inscritas en la razón moderno-occidental-colonial. Por eso, no se refiere a "otros modos", ni tampoco a "modos alternativos", sino a ellos asentados sobre las historias y experiencias de la diferencia colonial.

3 Este término se refiere a profesores que no realizan clases en la asignatura de mapuzungun y no son formados como profesores de educación intercultural bilingüe.

4 Este término alude a las asignaturas de lenguaje y comunicación, historia, geografía y ciencias sociales, ciencias naturales y matemática. 